\title{
Common gene haplotypes of gelatinases and their tissue inhibitors in abdominal aortic aneurysm
}

\author{
Mária Škereňová ${ }^{1,2}$, Tatiana Matáková1,3, Erika Halašová ${ }^{1,4}$, Lucia Rišková ${ }^{5}$, Michaela \\ Škorvanová ${ }^{3}$, Dušan Mištuna ${ }^{6}$, Mirko Halaša ${ }^{7}$ and Dušan Dobrota ${ }^{2,3}$ \\ ${ }^{1}$ Biomedical Center Martin, Department of Molecular Medicine, Jessenius Faculty of Medicine in Martin, Comenius University \\ in Bratislava, Martin, Slovakia \\ ${ }^{2}$ Department of Clinical Biochemistry, Jessenius Faculty of Medicine in Martin, Comenius University in Bratislava, Martin, \\ Slovakia \\ ${ }^{3}$ Department of Medical Biochemistry, Jessenius Faculty of Medicine in Martin, Comenius University in Bratislava, Martin, \\ Slovakia \\ ${ }^{4}$ Department of Medical Biology, Jessenius Faculty of Medicine in Martin, Comenius University in Bratislava, Martin, Slovakia \\ ${ }^{5}$ Hospital and Health Centre in Považská Bystrica, Považská Bystrica, Slovakia \\ ${ }^{6}$ Clinic of Surgery and Transplant Center, Jessenius Faculty of Medicine in Martin, Comenius University in Bratislava, Martin, \\ Slovakia \\ ${ }^{7}$ Clinic of Surgery and Transplant Center, University Hospital in Martin, Martin, Slovakia
}

\begin{abstract}
Abdominal aortic aneurysm (AAA) involves complex dynamic remodeling processes in the aortic wall. Gelatinases (MMP2 and MMP9) and their respective tissue inhibitors (TIMP1 and TIMP2) play a crucial role during extracellular matrix (ECM) turnover in aortic tissue. In this study we characterized associations between the haplotypes of genes encoding gelatinase/inhibitor pairs and pathways involved in AAA, a total of 100 AAA patients and 192 controls were enrolled. For males, a significant decrease in the distribution of the minor G allele of the TIMP2 rs8082025 was observed in AAA patients ( $p=0.01,23.1 \%$ controls $v s .13 .1 \%$ AAA). In addition, in males, the major TIMP2 GA haplotype was associated with AAA (86.9\% AAA vs. $76.9 \%$ control; $p=0.009$, $\mathrm{OR}=1.997)$, whereas the TIMP2 GG haplotype (7.7\% AAA vs. $13.9 \%$ control) was associated with protection against AAA ( $p=0.046, \mathrm{OR}=0.518)$. The minor GAGC MMP9 haplotype was related to AAA for all study subjects as well as the males only subset $(p=0.011, \mathrm{OR}=2.202$ and $p=0.025$, OR $=2.156$, respectively). Small differences in the distribution of gene haplotypes could be associated with different levels of gene expression and in turn influence gelatinases activity in AAA.
\end{abstract}

Key words: Abdominal aortic aneurysm - ECM - Gelatinases - MMP - TIMP - Haplotype

\section{Introduction}

Abdominal aortic aneurysm (AAA) is a complex dynamic remodeling process that occurs in the aortic wall. Vascular smooth muscle cell (VSMC) apoptosis, remodeling of the extracellular matrix (ECM), generation of elastic fibers, and

Correspondence to: Tatiana Matáková, Biomedical Center Martin, Department of Molecular Medicine, Jessenius Faculty of Medicine in Martin, Comenius University in Bratislava, Malá Hora 11161/4C Martin, Slovakia

E-mail: tatiana.matakova@uniba.sk destruction and inflammatory cell infiltration are all involved in AAA development (Guo et al. 2006). Aneurysmal tissue is rich in proteases that degrade the ECM as well as cytokines and chemokines that are produced by resident cells of the aortic wall or infiltrating immune cells. Aortic wall damage, chronic inflammatory reactions, and the presence of an intraluminal thrombus or infection can all serve as initiating events for aneurysm formation (Petersen et al. 2002; Michel et al. 2011; Shi and Lindholt 2013).

Matrix metalloproteinases (MMPs) play a crucial role in ECM turnover. MMPs belong to a family of zinc-dependent extracellular endopeptidases that have roles in many physi- 
ological (e.g., morphogenesis, tissue remodeling, embryonic development, cell growth, migration, proliferation and adhesion) and pathological processes, such as cancer, neurodegeneration, inflammation, and muscular dystrophy (Bozzi et al. 2015). The gelatinase group of the MMP family includes MMP2 and MMP9, which are important for aortic aneurysm pathogenesis. Gelatinase expression is increased at both the mRNA and protein level in human aneurysm tissues relative to normal aorta tissues (Davis et al. 1998). MMP2 (gelatinase A) is mainly produced by mesenchymal cells, whereas MMP9 (gelatinase B) is produced by infiltrating macrophages and neutrophils (Sakalihasan et al. 1996). A study by Longo et al. suggested that MMP expression by both mesenchymal cells and macrophages would be required for aneurysm formation (Longo et al. 2002).

Gelatinase expression and enzymatic activity is precisely regulated by the tissue inhibitors of metalloproteinases (TIMPs), which comprise a family of four endogenous proteins. Changes in MMP/TIMP ratios can modify the aortic wall structure and composition, and in turn promote aneurysm formation (Tamarina et al. 1997).

Variabilities in gene sequence or expression can contribute to MMP/TIMP imbalances in the aortic wall, although the extent of this contribution is unclear (Elefteriades et al. 2013). Two members of the TIMP family preferentially bind gelatinases: TIMP1 is an inhibitor of MMP9 and TIMP2 selectively inhibits MMP2. Previous studies examined the effect of sequence variations in gelatinase and TIMP genes, although the results were inconsistent (Hinterseher et al. 2007; Bradley et al. 2016). For TIMP2, variations in gene structure could be a factor in AAA, which is supported by the small proportion of $\mathrm{cDNA}(3 \%)$ relative to the entire gene sequence $(117 \mathrm{~kb})$. Thus, sequence variations in the regulatory regions of genes encoding MMPs and TIMPs may affect expression, whereas in the coding region, nonsynonymous variations could influence protein activity and function in relevant biological processes. Variability in target genomic regions can also be represented more accurately by common haplotypes, rather than by several single genotypes.

In this study, we examined the association between haplotype and AAA using the marked relationship between both gelatinase/inhibitor pairs at the phenotype level and their clear role in AAA pathogenesis.

\section{Materials and Methods}

\section{Study subjects}

A total of 100 patients with a main diagnosis of abdominal aortic aneurysm (AAA) with or without rupture (I71.3, I71.4, according to The International Classification of Diseases (ICD)) were included in the study. Each of the 100 AAA patients was the first person in their family (proband) to come to our attention. Included were all patients at the clinic who agreed and signed informed consent. The control group was assembled from 192 healthy, unrelated volunteers. All subjects were Caucasians of European origin. Healthy donors had no personal or family history of AAA. Written informed consent was obtained from all study subjects. This study was approved by the Human Subjects Committees of the Jessenius Faculty in Medicine in Martin at Comenius University, Bratislava. The study protocol conforms to the ethical guidelines of the 1975 Declaration of Helsinki.

In this study, we genotyped 100 AAA probands having an average age at diagnosis of $72.6 \pm 8.1$ years. The study cohort included 84 males (84\%) and 16 females (16\%). The control group included 192 healthy individuals (147 males (76.6\%) and 45 females (23.4\%)) with an average age of 57.3 \pm 10.5 years. Due to the predominance of males in this study sample, we also report a separate set of results for males only. Because of the minor allele frequency (MAF) greater than $10 \%$ we consider the sample size to be adequate to give enough power to detect common gene haplotypes.

The clinical characteristics for the AAA subjects were as follows: BMI $28.1 \pm 4.1$, current smokers $13 \%$, ex-smokers $62 \%$, hypertension $85 \%$, dyslipidemia $56 \%$, atherosclerosis $58 \%$, and peripheral vascular disease $57 \%$.

\section{Genes and tagged single nucleotide polymorphisms (tag SNPs)}

Basic characterization of the target genes is presented in Table 1. The data were generated with the 1000 Genomes

Table 1. Characteristics of study genes

\begin{tabular}{lccccccc}
\hline Gene & Transcript/Protein & GRCh38/hg38 & bp & Band & cDNA (bp) & AAs & Exons \\
\hline MMP2 & NM_001302508.1, NP_004521.1 & chr16:55,389,700-55,506,691 & 116,992 & $16 \mathrm{q} 12.2$ & 3,741 & 660 & 13 \\
TIMP2 & NM_003255.4, NP_003246.1 & chr17:78,852,977-78,925,390 & 72,414 & $17 \mathrm{q} 25.3$ & 3,652 & 220 & 5 \\
MMP9 & NM_004994.2, NP_004985.2 & chr20:46,008,908-46,016,561 & 7,654 & $20 \mathrm{q} 13.12$ & 2,336 & 707 & 13 \\
TIMP1 & NM_003254.2, NP_003245.1 & chrX:47,582,291-47,586,791 & 4,501 & Xp11.3 & 892 & 207 & 5 \\
\hline
\end{tabular}

GRCh, genome reference consortium human build; hg, human genome build; bp, base pair; Band, cytogenetic band; cDNA, coding DNA; AA, amino acid. 
Project (Phase 3) and an adopted algorithm available in Haploview 4.2 using CEU population, $\mathrm{a}^{2}$ threshold of $0.8, \mathrm{MAF}$ $>0.1$, and pair-wise tagging to select SNPs for tagging (Barrett et al. 2005; 1000 Genomes Project Consortium 2015).

Since the MMP2/TIMP2 gene pair is characterized by extensive gene loci, haplotype analysis for this pair focused on shorter regulatory regions.

Common variability (10 SNPs with MAF > 0.1) of the $M M P 2$ gene 5' regulatory region that covers 1,989 bases was captured by two tag SNPs (rs12599478 and rs2285053) with the nearGene- 5 function.

The annotation tracks of the UCSC Genome Browser were used to define regulatory signal regions of TIMP2 with conserved sequences located in the first intron of the longest transcript and the 5 ' regulatory region of the shorter transcripts. This region covers 2,833 bases and contains 18 intronic SNPs (MAF > 0.1) that were captured by two tagged SNPs (rs7342880 and rs8082025) and were selected for this study.

Four tag SNPs (rs2274755, rs17576, rs3787268, and rs20544) and one independent SNP (rs2250889) were used to capture common variability (22 SNPs with MAF > 0.1) within the entire $M M P 9$ gene that was expanded by $2 \mathrm{~kb}$ into the 5 ' regulatory region.

For the TIMP1 gene, three tag SNPs (rs35777532, rs4898, and rs6609534) were used to capture common variability (10 SNPs with MAF > 0.1) within the entire gene that was expanded by $1 \mathrm{~kb}$ into the 3 ' regulatory region.

References for tag SNPs as well as captured SNPs, positions, and locations in the human genome (assembly GRCh38.p7) and genotyping information are presented in Table 2.

\section{DNA analysis}

Whole blood samples were taken from each of the participants. Genomic DNA was extracted from $200 \mu \mathrm{l}$ whole blood using a MagNA Pure LC DNA Isolation Kit I (F. HoffmannLa Roche Ltd, Basel, Switzerland) on a MagNA Pure LC 2.0 (F. Hoffmann-La Roche Ltd, Basel, Switzerland) according to the manufacturer's instructions. The concentration of each DNA sample was set to $30 \mu \mathrm{g} / \mathrm{ml}$. DNA quantification was performed using an Invitrogen Qubit Fluorometer (Thermo Fisher Scientific Inc., Waltham, USA) and an Invitrogen Qubit dsDNA BR Assay Kit (Thermo Fisher Scientific Inc., Waltham, USA). High-resolution melting analysis (HRMA) was the main method used for genotyping of selected SNPs. A LightCycler 480 II, LightCycler 480 High Resolution Melting Master Mix and LightCycler 480 Gene Scanning Software V1.5.1 (all: F. Hoffmann La Roche Ltd, Basel, Switzerland) were used for HRMA. Primers (Table 2) for all genotyping reactions were designed using Primer3Plus software (Untergasser et al. 2012). The polymerase chain reaction-restriction fragment length polymorphism (PCR-RFLP) method was used to assess the rs2250889 genotype. The PCR-RFLP assay involved DreamTaq Green PCR Master Mix (2X) (Thermo Fisher Scientific Inc., Waltham, USA), modified primers and the restriction endonuclease FastDigest BamHI (Thermo Fisher Scientific Inc., Waltham, USA) (Table 2). Positive and negative controls were included in all reactions. Approximately $10 \%$ of all samples were used as blinded duplicates for "in-house" reaction quality control.

\section{Statistics}

Single marker analyses and haplotype analyses were conducted using SNP \& Variation Suite v8.3 (Golden Helix, Inc., Bozeman, MT, www.goldenhelix.com). Fisher's exact test was used to estimate the significance of deviation from Hardy-Weinberg equilibrium and to execute basic allelic associations. Pearson's chi-squared test for contingency tables was used to examine haplotype associations. A haplotype frequency was estimated using the EM algorithm. Association tests were confirmed by logistic regression with case/ control status as the dependent variable and suspected haplotype as the independent variable in a dominant genetic model. Logistic regressions were performed with the PASW statistical package in SPSS Statistics 18 (SPSS Inc., released 2009; PASW Statistics for Windows, Version 18.0. Chicago; SPSS, Inc.). Odds ratios (ORs) with 95\% confidence intervals $(95 \% \mathrm{CI})$ were used to assess genetic effects.

\section{Results}

Alleles

The observed genotype distributions for all tag SNPs were in Hardy-Weinberg equilibrium for both the AAA and control groups. Results for the allele frequency analysis of all targeted genetic variants are reported in Table 3. In our population, 3 of all 12 analyzed genetic variants had a MAF $<0.1$. For the gene pair MMP2/TIMP2, the MAF for all four genetic variants in the control group was slightly higher than that for the AAA group. For the TIMP2 rs8082025, there was a statistically significant difference in the MAF between the subgroups that included only male subjects (Table 3; 13.1\% AAA vs. $23.1 \%$ control; $p=0.01$, OR $(95 \% \mathrm{CI})=0.501$ $(0.297-0.846))$. At the second TIMP2 rs7342880 there was an apparent difference in the MAF between AAA patients and controls (Table 3; 5.4\% AAA vs. 9.2\% controls, respectively), although this difference did not achieve statistical significance. For the MMP9 rs2250889 coding variant c.1721G>C, p.Arg574Pro, an apparently higher OR (1.73) corresponding to an apparent difference in the MAF (Table 3; 7.0\% AAA vs. $4.2 \%$ control) was also not statistically significant. For all 


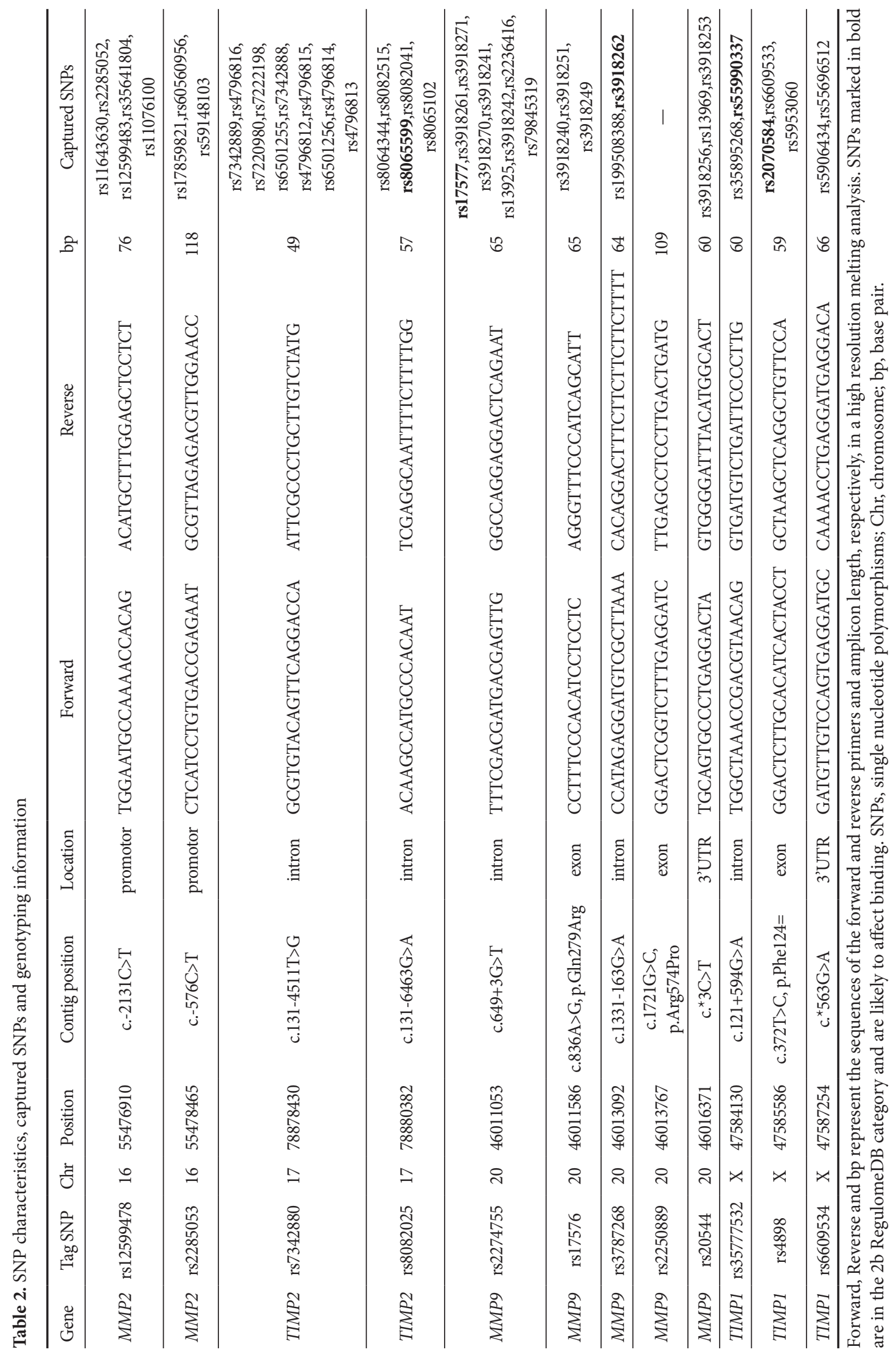


Table 3. Allele association analysis of all study subjects and for a subgroup that includes males only

\begin{tabular}{|c|c|c|c|c|c|c|c|c|c|c|}
\hline \multirow{4}{*}{$\begin{array}{l}\text { Gene } \\
\text { MMP2 }\end{array}$} & \multirow{4}{*}{$\begin{array}{c}\text { Marker } \\
\text { rs12599478 }\end{array}$} & \multirow{4}{*}{$\begin{array}{c}\text { Minor } \\
\text { Allele } \\
\text { C }\end{array}$} & \multicolumn{4}{|c|}{ Minor Allele Frequency } & \multirow{2}{*}{\multicolumn{2}{|c|}{ Fisher's Exact $p$}} & \multirow{2}{*}{\multicolumn{2}{|c|}{ OR $(95 \% \mathrm{CI})$}} \\
\hline & & & \multirow{2}{*}{$\begin{array}{c}\begin{array}{c}\text { AAA } \\
(n=100)\end{array} \\
\mathrm{al}\end{array}$} & \multirow{2}{*}{$\begin{array}{l}\text { Controls } \\
(n=192) \\
11\end{array}$} & \multirow{2}{*}{$\begin{array}{r}\begin{array}{c}\text { AAA } \\
(n=84)\end{array} \\
\mathrm{ma}\end{array}$} & \multirow{2}{*}{$\begin{array}{l}\text { Controls } \\
(n=147) \\
\text { les }\end{array}$} & & & & \\
\hline & & & & & & & all & males & all & males \\
\hline & & & 0.360 & 0.385 & 0.357 & 0.378 & 0.590 & 0.690 & $0.897(0.629-1.278)$ & $0.916(0.618-1.358)$ \\
\hline$M M P 2$ & rs2285053 & $\mathrm{T}$ & 0.115 & 0.120 & 0.113 & 0.122 & 0.894 & 0.881 & $0.955(0.561-1.626)$ & $0.914(0.506-1.651)$ \\
\hline TIMP2 & rs7342880 & $\mathrm{T}$ & 0.060 & 0.086 & 0.054 & 0.092 & 0.327 & 0.153 & $0.679(0.343-1.346)$ & $0.56(0.257-1.221)$ \\
\hline TIMP2 & rs8082025 & G & 0.155 & 0.216 & 0.131 & 0.231 & 0.079 & 0.010 & $0.665(0.423-1.047)$ & $0.501(0.297-0.846)$ \\
\hline MMP9 & rs2274755 & $\mathrm{T}$ & 0.150 & 0.156 & 0.137 & 0.156 & 0.904 & 0.591 & $0.953(0.592-1.534)$ & $0.855(0.498-1.469)$ \\
\hline MMP9 & rs17576 & G & 0.330 & 0.372 & 0.339 & 0.374 & 0.319 & 0.482 & $0.83(0.579-1.19)$ & $0.859(0.577-1.278)$ \\
\hline MMP9 & rs3787268 & A & 0.180 & 0.216 & 0.202 & 0.218 & 0.331 & 0.724 & $0.796(0.515-1.23)$ & $0.912(0.571-1.455)$ \\
\hline MMP9 & rs2250889 & G & 0.070 & 0.042 & 0.071 & 0.041 & 0.167 & 0.191 & $1.731(0.827-3.624)$ & $1.808(0.793-4.12)$ \\
\hline MMP9 & rs20544 & $\mathrm{C}$ & 0.445 & 0.427 & 0.458 & 0.432 & 0.725 & 0.627 & $1.076(0.762-1.518)$ & $1.113(0.76-1.629)$ \\
\hline TIMP1 & rs35777532 & A & - & - & 0.071 & 0.061 & - & 0.697 & - & $1.179(0.554-2.513)$ \\
\hline TIMP1 & rs4898 & $\mathrm{T}$ & - & - & 0.458 & 0.493 & - & 0.499 & - & $0.869(0.595-1.271)$ \\
\hline TIMP1 & rs6609534 & A & - & - & 0.369 & 0.415 & - & 0.374 & - & $0.825(0.558-1.218)$ \\
\hline
\end{tabular}

AAA, abdominal aortic aneurysm; OR, odds ratio; CI, confidence interval; $n$, number of individuals per group; bold font indicates statistical significance. (The X chromosome TIMP1 allele frequency is reported only for males).

other variants the independence of examined groups and alleles was confirmed by Fisher's exact test.

\section{Haplotypes}

Haplotype analysis was performed to further evaluate the role of the tested genes in AAA susceptibility. Three common haplotypes having a sample frequency above 0.01 were estimated for the regulatory regions of the $M M P 2$ and TIMP2 genes as defined by a pair of tag SNPs. Five and four common haplotypes were estimated for MMP9 (5 tag SNPs) and TIMP1 (3 tag SNPs) genes, respectively. MMP9 rs2250889 (the fourth position in the haplotype) contributed to the gene haplotype variability by splitting up the rarest haplotype (gaggc and gagcc) only. Since all other MMP9 haplotypes have a $\mathrm{C}$ allele at the fourth position, MMP9 haplotypes were re-estimated without rs2250889. Four common haplotypes were estimated for both MMP9 and TIMP1 genes (Table 4).

The MMP2 haplotype frequencies were similar between AAA and the controls in both the complete sample and the subgroup that included only males, and no significant associations were detected. The tendency of the TIMP2 haplotype distribution between AAA and controls for all study subjects was supported by the significant differences that were observed when only males were considered. For the subgroup that included males only, the major TIMP2 GA haplotype (Table 4; 86.9\% AAA vs. 76.9\% controls) was significantly associated with AAA $(p=0.009$, OR (95\%CI) $=1.997$ (1.183-3.372)), whereas the second most common TIMP2 GG haplotype (Table 4; 7.7\% AAA vs. 13.9\% controls) was significantly associated with protection against AAA $(p=0.046$, OR $(95 \% \mathrm{CI})=0.518(0.269-0.996))$. The MMP9 haplotype frequencies were similar between AAA patients and the controls except for the minor GAGC haplotype, which was significantly associated with AAA both for the group with all subjects as well as the males only subgroup (Total: $p=0.011$, OR $(95 \% \mathrm{CI})=2.202(1.180-4.109)$; Males only: $p=0.025$, OR $(95 \% \mathrm{CI})=2.156(1.089-4.269))$. The TIMP1 haplotype frequencies were similar between AAA patients and the control subjects, and approximately $90 \%$ of the samples were covered by the two major GTG and GCA haplotypes. No significant associations between AAA and haplotype were detected.

The significant differences for TIMP2 and MMP9 haplotypes were confirmed by logistic regression.

\section{Interactions}

The distributions of the combined diplotypes between gelatinases and their tissue inhibitor counterparts in the subgroup that included males only are shown in Table 5.

For $M M P 2$, more than $65 \%$ of the samples could be described by only four categories for both TIMP1 and TIMP2 interactions. The frequency of the category "TC/CC MMP2 \& GA/GA TIMP2", with two possible interactions, TC_GA 
Table 4. Haplotype association analysis of all study subjects and for male subjects only

\begin{tabular}{|c|c|c|c|c|c|c|c|c|c|c|c|}
\hline \multirow{2}{*}{ Gene } & \multirow{2}{*}{ Haplotype } & \multicolumn{5}{|c|}{ all } & \multicolumn{5}{|c|}{ males } \\
\hline & & AAA & controls & $\mathrm{P}$ (chi2) & chi2 & OR (95\%CI) & AAA & controls & $\mathrm{P}$ (chi2) & chi2 & OR $(95 \% \mathrm{CI})$ \\
\hline \multirow{3}{*}{ MMP2 } & TC & 0.639 & 0.608 & 0.516 & 0.422 & $1.125(0.789-1.604)$ & 0.641 & 0.614 & 0.622 & 0.243 & $1.104(0.744-1.638)$ \\
\hline & $\mathrm{CC}$ & 0.246 & 0.272 & 0.476 & 0.509 & $0.867(0.585-1.284)$ & 0.245 & 0.263 & 0.638 & 0.221 & $0.901(0.582-1.394)$ \\
\hline & $\mathrm{CT}$ & 0.114 & 0.113 & 0.999 & 0.000 & $1(0.583-1.713)$ & 0.112 & 0.114 & 0.918 & 0.011 & $0.969(0.532-1.765)$ \\
\hline \multirow{3}{*}{ TIMP2 } & GA & 0.845 & 0.784 & 0.077 & 3.130 & $1.503(0.955-2.366)$ & 0.869 & 0.769 & 0.009 & 6.862 & $1.997(1.183-3.372)$ \\
\hline & GG & 0.095 & 0.130 & 0.211 & 1.565 & $0.701(0.401-1.226)$ & 0.077 & 0.139 & 0.046 & 3.991 & $0.518(0.269-0.996)$ \\
\hline & TG & 0.060 & 0.086 & 0.265 & 1.244 & $0.679(0.343-1.346)$ & 0.054 & 0.092 & 0.140 & 2.179 & $0.56(0.257-1.221)$ \\
\hline \multirow{6}{*}{ MMP9 } & GAGCT & 0.549 & 0.573 & 0.775 & 0.082 & $0.951(0.672-1.345)$ & 0.535 & 0.568 & 0.676 & 0.175 & $0.921(0.627-1.353)$ \\
\hline & GGACC & 0.175 & 0.216 & 0.288 & 1.130 & $0.788(0.508-1.223)$ & 0.196 & 0.218 & 0.679 & 0.171 & $0.905(0.565-1.451)$ \\
\hline & TGGCC & 0.145 & 0.156 & 0.793 & 0.069 & $0.938(0.58-1.517)$ & 0.131 & 0.156 & 0.520 & 0.415 & $0.835(0.483-1.445)$ \\
\hline & $\mathrm{GAG}(\mathrm{C} / \mathrm{G}) \mathrm{C}$ & 0.111 & 0.055 & 0.011 & 6.408 & $2.202(1.18-4.109)$ & 0.114 & 0.058 & 0.025 & 5.043 & $2.156(1.089-4.269)$ \\
\hline & GAGGC & 0.070 & 0.042 & 0.126 & 2.343 & $1.769(0.845-3.704)$ & 0.071 & 0.041 & 0.136 & 2.219 & $1.855(0.814-4.23)$ \\
\hline & GAGCC & 0.040 & 0.013 & 0.031 & 4.631 & $3.243(1.048-10.038)$ & 0.042 & 0.017 & 0.096 & 2.773 & $2.596(0.812-8.301)$ \\
\hline \multirow{4}{*}{ TIMP1 } & GTG & 0.530 & 0.492 & 0.324 & 0.973 & $1.189(0.843-1.676)$ & 0.542 & 0.507 & 0.393 & 0.728 & $1.181(0.806-1.73)$ \\
\hline & GCA & 0.375 & 0.406 & 0.522 & 0.411 & $0.891(0.627-1.267)$ & 0.357 & 0.415 & 0.260 & 1.271 & $0.798(0.539-1.182)$ \\
\hline & ACG & 0.055 & 0.073 & 0.428 & 0.627 & $0.748(0.364-1.537)$ & 0.060 & 0.061 & 0.966 & 0.002 & $0.983(0.443-2.182)$ \\
\hline & GCG & 0.030 & 0.029 & 0.913 & 0.012 & $1.058(0.386-2.903)$ & 0.030 & 0.017 & 0.354 & 0.858 & $1.795(0.512-6.294)$ \\
\hline
\end{tabular}

SNP order in haplotypes: MMP2 - rs12599478, rs2285053; TIMP2 - rs7342880, rs8082025; MMP9 - rs2274755, rs17576, rs3787268, rs20544; TIMP1 - rs35777532, rs4898, rs6609534. Italic font indicates haplotypes resulted from splitting of GAGC by rs2250889, which is located between the third and fourth haplotype position. Bold font indicates statistical significance. AAA, abdominal aortic aneurysm; OR, odds ratio; CI, confidence interval.

or CC_GA, showed a marked decrease for the control group (25.6\% AAA vs. $16.4 \%$ control). In contrast, the category “TC/CC MMP2 \& GA/GG TIMP2" showed a marked decrease relative to controls ( $2.4 \%$ AAA $v s .8 .9 \%$ control). The majority of MMP2 and TIMP1 interaction categories showed no significant differences between samples.

MMP9 exhibited a range of distributions for both TIMP1 and TIMP2 interactions. Only the GAGT/GAGC MMP9 diplotype with the risk GAGC MMP9 haplotype fell into categories that showed noticeable differences. The category "GAGT/GAGC MMP9 \& GA/GA TIMP2" with GAGT_GA or GAGC_GA interactions showed a marked increase for AAA relative to controls ( $12.2 \%$ vs. 3.4\%, respectively) and the category "GAGT/GAGC MMP9 \& GTG TIMP1" with GAGT_GTG or GAGC_GTG interactions was increased for AAA patients compared to controls (7.3\% vs. 2.1\%, respectively).

\section{RegulomeDB binding scores}

RegulomeDB is a database that annotates SNPs with known and predicted regulatory elements in the intergenic regions of the human genome (Boyle et al. 2012). Given the size of the MMP2/TIMP2 gene pair, only selected regulatory regions of the genes were analyzed, such that all tagged and captured
SNPs were in introns or promoter regions (Table 2). The RegulomeDB binding score for these SNPs ranged from 4 to 6 , with only TIMP2 rs8065599 captured by tag rs 8082025 scoring as $2 \mathrm{~b}$, suggesting a possible effect on binding.

For the MMP9/TIMP1 gene pair, the common variability of the entire gene sequence was analyzed. The RegulomeDB binding score for the tagged and captured SNPs for this gene pair ranged predominantly from 4 to 6. One MMP9SNP was scored as 3a, and thus was predicted to be less likely to affect binding, whereas rs 17577 and rs3918262 both scored 2b. For TIMP1, four SNPs were scored as 3a, and two, rs55990337 and rs2070584, were scored as $2 \mathrm{~b}$.

With the exception of $M M P 2$, tag SNPs for all of the studied genes captured variants in sequence motifs that have a regulatory function.

\section{Discussion}

AAA occurs as a hereditary or acquired disease. Male gender and increasing age are strong risk factors for AAA. In addition, individuals with a history of smoking, hypertension, dyslipidemia, and other medical conditions, such as coronary heart disease and peripheral vascular disease, have an increased risk of developing AAA (Golledge et al. 2006). One 
study examining twins showed that in $70 \%$ of cases, both twins had AAA (Wahlgren et al. 2010). However, there are currently no definitive theories on the underlying genetic mechanisms of this multifactorial disease. Some association studies and genome-wide association studies have identified genes that are suspected to have an association with increased AAA risk (Bown et al. 2011; Bradley et al. 2013; van 't Hof et al. 2016; Jones et al. 2017).

Matrix metalloproteinases and tissue inhibitors of metalloproteinases play an important role in the formation of AAA (Longo et al. 2002). Among the MMPs, the gelatinase subgroup is unique in that it has two members that contain fibronectin type II domains (FN2) within the catalytic domains. These FN2 domains allow for MMP2 and MMP9 to degrade ECM constituents, including type IV collagen, type $\mathrm{V}$ collagen, all types of denatured collagens (e.g., gelatins), and elastin (Shi et al. 2012).

Endogenous tissue inhibitors of matrix metalloproteinases bind MMPs in a 1:1 stoichiometry; however, due to the lack of binding specificity, multiple tissue-specific interactions are part of local proteolytic activity networks (Visse and Nagase 2003).

This study sought to describe common variabilities in regions of two gelatinase genes (MMP2 and MMP9) and genes encoding their counterparts, tissue inhibitors of matrix metalloproteinases (TIMP1 and TIMP2). In addition, associations of observed haplotypes in patients with phenotypic expression of AAA were modeled and the haplotype interactions were categorized.

A protective trend for two minor TIMP2 haplotypes was seen for the subgroup that included males only. The minor TIMP2 haplotypes, GG and TG, differed in alleles for rs7342880, whereas rs8082025 had the same G allele. The GG TIMP2 haplotype had protective potential against AAA ( $p=$ $0.009)$. In the first intron in the regulatory signal region of the conserved sequences, approximately $80 \%$ of the study population had the same major GA haplotype.

In AAA patients, the MMP9 haplotype GAGC occurred twice as often as in controls ( $11.1 \%$ vs. $5.5 \%$, respectively). Relative to the most common haplotype differs only in the last position matched to the variation in the 3' UTR, which is a target of regulatory molecules. Moreover, GAGC is the genetic background for the minor allele of the coding variation rs2250889 in exon 10 and results in a change from Arg to Pro at amino acid 574, which lies in the second hemopexin repeat domain of MMP9. The G allele was transmitted via GAGC haplotype. Because proline is the only cyclic nonpolar amino acid and arginine carries a positive charge, this substitution may produce substantial changes that could manifest as structural differences or affect electrostatic interactions with binding partners.

The next coding variation in $M M P 9$, rs17577, was captured by the tag SNP rs2274755. The minor allele is inherited only on the genetic background of the TGGC haplotype. This variation occurs in exon 12 and results in a change from arginine to glutamine at position 668 in the fourth hemopexin repeat domain of MMP9 and is essentially a conservative amino acid change in terms of polarity and structure. Thus, this variant can likely be tolerated and would not substantially affect the protein function.

The common variation rs17576 (c.836A>G, p.Gln279Arg) is found within the second highly conserved gelatinasespecific FN2, and presumably enhances substrate binding (Cong et al. 2009). The higher MAF (> 35\%) and the 50\% rate for heterozygotes seen among the control subjects suggests that variability in this amino acid position can be tolerated or is desirable in certain contexts.

The minor G allele of rs3918262 captured by the tag SNP rs3787268 allele A is inherited only on the genetic background of the GGAC haplotype. The variation rs3918262 (c.2005+657A $>\mathrm{G}$ ) located in the last intron is "likely to affect binding", according to RegulomeDB, and according to the GTEx Portal is linked to single-tissue (adipose, lung, aorta) expression quantitative trait loci (eQTLs), but not MMP9 gene loci.

The TIMP1 haplotypes on the $\mathrm{X}$ chromosome, which has only one copy in males, did not show significant differences in frequency between AAA patients and controls. These haplotypes can be linked to TIMP1 expression levels in aortic tissues due to linkage disequilibrium with variations that are "likely to affect binding", but their effects await further exploration. The minor GCG haplotype had an approximately 1.8-fold higher likelihood of association with AAA, but this relationship did not achieve statistical significance.

In the interaction analysis, we focused on variabilities in the population of male subjects in possible combination with variations in gelatinase and their tissue inhibitor haplotypes. Regulatory region haplotypes in MMP2 and TIMP2 showed a shift in the most common combination between controls and AAA. The pure combination TC MMP2 and GA TIMP2 haplotypes (TCxGA) occurred at a rate of approximately $30 \%$ in both groups.

On the major TCxGA background, both CC MMP2 in combination with GA TIMP2 and TC MMP2 in combination with GG TIMP2 were more frequent in AAA patients compared to control subjects $(25.6 \%$ vs. $16.4 \%$ and $8.5 \%$ vs. $4.8 \%$, respectively). Although the simultaneous presence of four varied haplotypes was more frequent in the controls than in AAA patients ( $8.9 \%$ vs. $2.4 \%$, respectively), the MMP2 haplotypes showed no differences in distribution between AAA and controls. In fact, in combination with TIMP2 haplotypes, we noticed potential risk combinations for CCxGA and TCxGG on the major TCxGA background. Differences in the other haplotype combinations also were not remarkable, except for the risk of AAA associated with the GAGC MMP9 haplotype. Patients carrying the major 
GA TIMP2 haplotype had an increased risk of AAA $(\mathrm{OR}=$ 3.917), whereas there was no significant difference in risk between carriers of the major GTG TIMP1 haplotype and non-carriers $(\mathrm{OR}=3.763)$ (Table 5).

Of the SNPs analyzed in the present study, rs2285053 (MMP2), rs17576 rs3918242, rs17577, rs2250889 (MMP9), and rs4898 (TIMP1) are the most frequently studied, as evidenced by the $111,136,430,48,45$, and 41 entries, respectively, in LitVar (Wei et al. 2018). Genetic variants in these SNPs are associated with numerous human diseases, and many of these studies examined the relationship between disease pathology and gelatinase and TIMP function. The most frequently documented diseases were coronary artery disease, neoplasms, cancers, hypertension, endometriosis, glaucoma, and aortic aneurysms. More than ten publications describe an association analysis of MMP9 rs3918242 (in full linkage disequilibrium (LD) with rs2274755 from the present study) with AAA, but the results of these studies were discordant (Lamblin et al. 2002; Jones et al. 2003; Powell 2006; Smallwood et al. 2008; Crkvenac Gregorek et al. 2016). Duellmann et al. documented the importance of $M M P 9$ genetic polymorphisms in progression of AAA (Duellman et al. 2012, 2014). In addition, several extensive reviews and meta-analyses of the relationship between AAA and variants of various genes, including metalloproteinases and TIMPs, have been published (Thompson et al. 2008; Yasmin and O'Shaughnessy 2008; Krishna et al. 2010; Morris et al. 2014; Bradley et al. 2016; Li et al. 2018).

Crkvenac Gregorek et al. failed to detect in Croatian patients with AAA the difference regarding MMP9 poly- morphism, except when the adjusted recessive model was applied (Crkvenac Gregorek et al. 2016).

The largest meta-GWAS of AAA performed by Jones et al. confirmed the central role for MMP9 in AAA with identifying direct interactions between ERG and MMP9 (Jones et al. 2003). The proxySNP near the MMP9 gene region showed a strong signal but is not in linkage with rs3918242.

Mikołajczyk-Stecyna et al. aimed to determine the relationship between the rs 8179090 polymorphism in the promotor region of the TIMP2 gene and AAA in Polish patients (Mikołajczyk-Stecyna et al. 2015). Their study indicated that the polymorphism is a risk factor of AAA with the highest OR for the male subpopulation.

Ogata et al. investigated 14 polymorphisms in 13 candidate genes (included MMP2, MMP9, TIMP1 and TIMP2) in multi-population study (Ogata et al. 2005). Analyses showed an association with the two TIMP1 gene polymorphisms in male AAA patients that remained significant when analyzing TIMP1 haplotypes. They concluded that the genetic variations in TIMP1 may contributed to the pathogenesis of AAA.

Smallwood et al. aimed to explore the association between potentially functional variants in the promotor region of the MMP9 gene and AAA in the Australian male population (Smallwood et al. 2008). No evidence of an association between two putatively functional polymorphisms of the MMP9 gene, or the most common gene haplotype, and AAA males has found in this study, which used an age-matched controls who have all had an ultrasound scan to exclude an AAA. As well, nonsignificant trend towards higher serum

Table 5. Percentage distribution of interactions between gelatinase and tissue inhibitor haplotypes in males

\begin{tabular}{|c|c|c|c|c|c|c|c|c|c|c|c|c|}
\hline & \multicolumn{6}{|c|}{$d_{-}$TIMP2 } & \multicolumn{6}{|c|}{ TIMP1 } \\
\hline & \multicolumn{2}{|c|}{ GA/GA } & \multicolumn{2}{|c|}{ GA/GG } & \multicolumn{2}{|c|}{ GA/TG } & \multicolumn{2}{|c|}{ GTG } & \multicolumn{2}{|c|}{ GCA } & \multicolumn{2}{|c|}{ ACG } \\
\hline & $\begin{array}{l}\text { AAA } \\
(\%)\end{array}$ & $\begin{array}{c}\text { Controls } \\
(\%)\end{array}$ & $\begin{array}{c}\text { AAA } \\
(\%)\end{array}$ & $\begin{array}{c}\text { Controls } \\
(\%)\end{array}$ & $\begin{array}{c}\mathrm{AAA} \\
(\%)\end{array}$ & $\begin{array}{c}\text { Controls } \\
(\%)\end{array}$ & $\begin{array}{l}\text { AAA } \\
(\%)\end{array}$ & $\begin{array}{c}\text { Controls } \\
(\%)\end{array}$ & $\begin{array}{c}\text { AAA } \\
(\%)\end{array}$ & $\begin{array}{c}\text { Controls } \\
(\%)\end{array}$ & $\begin{array}{c}\mathrm{AAA} \\
(\%)\end{array}$ & $\begin{array}{c}\text { Controls } \\
(\%)\end{array}$ \\
\hline \multicolumn{13}{|l|}{ d_MMP2 } \\
\hline $\mathrm{TC} / \mathrm{TC}$ & 29.3 & 28.1 & 8.5 & 4.8 & 3.7 & $4.1)$ & 25.6 & 19.2 & 12.2 & 17.1 & 2.4 & 2.1 \\
\hline $\mathrm{TC} / \mathrm{CC}$ & 25.6 & 16.4 & 2.4 & 8.9 & 6.1 & 2.7 & 15.9 & 14.4 & 13.4 & 13.0 & 2.4 & 2.7 \\
\hline $\mathrm{TC} / \mathrm{CT}$ & 7.3 & 8.9 & 4.9 & 4.1 & 0.0 & 0.7 & 7.3 & 10.3 & 4.9 & 4.1 & 0.0 & 0.7 \\
\hline $\mathrm{CC} / \mathrm{CC}$ & 3.7 & 2.1 & 0.0 & 3.4 & 1.2 & 2.7 & 2.4 & 3.4 & 2.4 & 4.1 & 0.0 & 0.0 \\
\hline $\mathrm{CT} / \mathrm{CT}$ & 2.4 & 1.4 & 0.0 & 0.0 & 0.0 & $0.0)$ & 0.0 & 1.4 & 2.4 & 0.0 & 0.0 & 0.0 \\
\hline $\mathrm{CC} / \mathrm{CT}$ & 4.9 & 2.7 & 0.0 & 1.4 & 0.0 & 0.7 & 3.7 & 1.4 & 0.0 & 3.4 & 1.2 & 0.7 \\
\hline \multicolumn{13}{|l|}{ d_MMP9 } \\
\hline GAGT/GAGT & 15.9 & 19.2 & 7.3 & 6.9 & 2.4 & 3.4 & 14.6 & 17.8 & 8.5 & 11.6 & 2.4 & 2.1 \\
\hline GAGT/GGAC & 19.5 & 16.4 & 1.2 & 2.7 & 2.4 & 2.1 & 13.4 & 11.0 & 8.5 & 10.3 & 0.0 & 1.4 \\
\hline GAGT/GAGC & 12.2 & $3.4^{\mathrm{a}}$ & 2.4 & 3.4 & 1.2 & 0.7 & 7.3 & $2.1^{b}$ & 6.1 & 5.5 & 1.2 & 0.7 \\
\hline GAGT/TGGC & 9.8 & 9.6 & 0.0 & 5.5 & 3.7 & 3.4 & 6.1 & 11.6 & 6.1 & 6.8 & 1.2 & 0.0 \\
\hline GGAC/TGGC & 8.5 & 4.1 & 1.2 & 2.1 & 0.0 & 0.7 & 6.1 & 3.4 & 2.4 & 3.4 & 1.2 & 0.7 \\
\hline GGAC/GGAC & 2.4 & 4.8 & 1.2 & 0.7 & 0.0 & 0.7 & 3.7 & 2.7 & 0.0 & 2.1 & 0.0 & 1.4 \\
\hline
\end{tabular}

AAA, abdominal aortic aneurysm; $\mathrm{d}_{-}$, diplotype prefix; ${ }^{\mathrm{a}} \mathrm{OR}=3.917{ }^{\mathrm{b}} \mathrm{OR}=3.763$; bold font indicates remarkable differences. 
levels of MMP9 in the minor allele carriers for both SNPs in males with AAA has found.

We are not aware of the existence of a study in AAA patients with an ambition to analyze the common variability of such genes or gene regions as present study offers. Our study supports the findings that common variants in the MMP9 gene are involved in the early stages of AAA formation via MMP/TIMP-mediated elastin degradation pathways (Thompson et al. 2002). The risk of AAA associated with the GAGC haplotype could be due to the genetic background of the deleterious coding mutation, or could be linked with regulators of gene expression or post-transcriptional events.

Our study revealed low variability in the little-studied conserved regulatory region of the TIMP2 gene. Here we showed that phenotypes of the major GA haplotype interact with MMP counterparts in AAA and that minor haplotypes have a protective function. According to our results, combinations of the GAGC haplotype with major TIMP1 or TIMP2 haplotypes are preferred for a higher risk of AAA.

Some tag SNPs and estimated haplotypes are in LD with sequence motif variations that may drive binding of transcription factors and regulatory molecules, such as hormones and inflammatory cytokines. Our results indicate that, in general, small differences in the distribution of $M M P 2$, MMP9, TIMP1, and TIMP2 haplotypes between AAA and controls can be highlighted at the level of gene expression. Recent studies suggest that modifications to the expression patterns of MMP and TIMP could occur in AAA and normal aortic tissues (Tamarina et al. 1997; Higashikata et al. 2004; Klaus et al. 2017). Additional studies are needed to validate the connections between commonly occurring genes or common regulatory haplotypes and the transcription levels of gelatinases and their tissue inhibitors.

\section{Study limitations}

There are two major limitations in this study that could be addressed in future research. First, the study did not have the power to detect small differences in estimated haplotype frequencies and haplotype interactions between groups, the sample size was too small to perform meaningful subanalyses. Second, the study may suffer from sampling bias since the participants in our study were not a random population sample. In a given time period of the sample collecting, all patients at the clinic who met the criteria and agreed and signed informed consent were included. The results should be interpreted in a careful manner.

Acknowledgement. This work was supported by "Center of translational medicine”, ITMS:26220220021 as well as "Center of excellency for research in personalized therapy", ITMS:26220120053, which are co-funded from EU sources and the European Regional Develop- ment Fund. The Genotype-Tissue Expression (GTEx) Project was supported by the Common Fund of the Office of the Director of the National Institutes of Health, and by NCI, NHGRI, NHLBI, NIDA, $\mathrm{NIMH}$, and NINDS. The data used for the analyses described in this manuscript were obtained from the GTEx Portal on 05/02/2018.

Conflict of interest. No actual or potential conflict of interest including any financial, personal or other relationships with other people or organizations exist.

\section{References}

1000 Genomes Project Consortium (2015): A global reference for human genetic variation. Nature 526, 68-74 https://doi.org/10.1038/nature15393

Barrett JC, Fry B, Maller J, Daly MJ (2005): Haploview: analysis and visualization of $\mathrm{LD}$ and haplotype maps. Bioinformatics 21, 263-265

https://doi.org/10.1093/bioinformatics/bth457

Bown MJ, Jones GT, Harrison SC, Wright BJ, Bumpstead S, Baas AF, Gretarsdottir S, Badger SA, Bradley DT, Burnand K, et al. (2011): Abdominal aortic aneurysm is associated with a variant in low-density lipoprotein receptor-related protein 1 . Am. J. Hum. Genet. 89, 619-627 https://doi.org/10.1016/j.ajhg.2011.10.002

Boyle AP, Hong EL, Hariharan M, Cheng Y, Schaub MA, Kasowski M, Karczewski KJ, Park J, Hitz BC, Weng S, et al. (2012): Annotation of functional variation in personal genomes using RegulomeDB. Genome Res. 22, 1790-1797 https://doi.org/10.1101/gr.137323.112

Bozzi M, Sciandra F, Brancaccio A (2015): Role of gelatinases in pathological and physiological processes involving the dystrophin-glycoprotein complex. Matrix Biology 44-46, 130-137 https://doi.org/10.1016/j.matbio.2015.02.005

Bradley DT, Hughes AE, Badger SA, Jones GT, Harrison SC, Wright BJ, Bumpstead S, Baas AF, Grétarsdóttir S, Burnand K, et al. (2013): A variant in LDLR is associated with abdominal aortic aneurysm. Circ. Cardiovasc. Genet. 6, 498-504 https://doi.org/10.1161/CIRCGENETICS.113.000165

Bradley DT, Badger SA, McFarland M, Hughes AE (2016): Abdominal aortic aneurysm genetic associations: mostly false? A systematic review and meta-analysis. Eur. J. Vasc. Endovasc. Surg. 51, 64-75 https://doi.org/10.1016/j.ejvs.2015.09.006

Cong Y, Guo X, Liu X, Cao D, Jia X, Xiao X, Li S, Fang S, Zhang Q (2009): Association of the single nucleotide polymorphisms in the extracellular matrix metalloprotease-9 gene with PACG in southern China. Mol. Vis. 15, 1412-1417

Crkvenac Gregorek A, Gornik KC, Polancec DS, Dabelic S (2016): Association of $1166 \mathrm{~A}>\mathrm{C}$ AT1R, $-1562 \mathrm{C}>\mathrm{T}$ MMP-9, ACE $\mathrm{I} / \mathrm{D}$, and CCR5 $\Delta 32$ polymorphisms with abdominal aortic aneurysm in croatian patients. genet. test. Mol. Biomarkers 20, 616-623 https://doi.org/10.1089/gtmb.2016.0158

Davis V, Persidskaia R, Baca-Regen L, Itoh Y, Nagase H, Persidsky Y, Ghorpade A, Baxter BT. (1998): Matrix metalloproteinase-2 
production and its binding to the matrix are increased in abdominal aortic aneurysms. Arterioscler. Thromb. Vasc. Biol. 18, 1625-1633 https://doi.org/10.1161/01.ATV.18.10.1625

Duellman T, Warren CL, Peissig P, Wynn M, Yang J (2012): Matrix metalloproteinase- 9 genotype as a potential genetic marker for abdominal aortic aneurysm. Circ. Cardiovasc. Genet. 5, 529-537 https://doi.org/10.1161/CIRCGENETICS.112.963082

Duellman T, Warren CL, Matsumura J, Yang J (2014): Analysis of multiple genetic polymorphisms in aggressive-growing and slow-growing abdominal aortic aneurysms. J. Vasc. Surg. 60, 613-621, e3 https://doi.org/10.1016/j.jvs.2014.03.274

Elefteriades JA, Habel N, Sun W, Sang AX, Kuzmik GA (2013): The aortic wall: four questions and insights. J. Thorac. Cardiovasc. Surg. (Suppl. 3) 145, S130-134 https://doi.org/10.1016/j.jtcvs.2012.11.050

Golledge J, Muller J, Daugherty A, Norman P (2006): Abdominal aortic aneurysm: pathogenesis and implications for management. Arterioscler. Thromb. Vasc. Biol. 26, 2605-2613 https://doi.org/10.1161/01.ATV.0000245819.32762.cb

Guo D-C, Papke CL, He R, Milewicz DM (2006): Pathogenesis of thoracic and abdominal aortic aneurysms. Ann. NY Acad. Sci. 1085, 339-352 https://doi.org/10.1196/annals.1383.013

Higashikata T, Yamagishi M, Sasaki H, Minatoya K, Ogino H, Ishibashi-Ueda H, Hao H, Nagaya N, Tomoike H, Sakamoto A (2004): Application of real-time RT-PCR to quantifying gene expression of matrix metalloproteinases and tissue inhibitors of metalloproteinases in human abdominal aortic aneurysm. Atherosclerosis 177, 353-360 https://doi.org/10.1016/j.atherosclerosis.2004.07.013

Hinterseher I, Krex D, Kuhlisch E, Schmidt KG, Pilarsky C, Schneiders W, Saeger HD, Bergert H (2007): Tissue inhibitor of metalloproteinase-1 (TIMP-1) polymorphisms in a Caucasian population with abdominal aortic aneurysm. World J. Surg. 31, 2248-2254 https://doi.org/10.1007/s00268-007-9209-X

Jones GT, Phillips VL, Harris EL, Rossaak JI, van Rij AM (2003): Functional matrix metalloproteinase-9 polymorphism (C$1562 \mathrm{~T}$ ) associated with abdominal aortic aneurysm. J. Vasc. Surg. 38, 1363-1367 https://doi.org/10.1016/S0741-5214(03)01027-9

Jones GT, Tromp G, Kuivaniemi H, Gretarsdottir S, Baas AF, Giusti B, Strauss E, Van't Hof FN, Webb TR, Erdman R, et al. (2017): Meta-analysis of genome-wide association studies for abdominal aortic aneurysm identifies four new disease-specific risk loci. Circ. Res. 120, 341-353

Klaus V, Tanios-Schmies F, Reeps C, Trenner M, Matevossian E, Eckstein $\mathrm{HH}$, Pelisek J (2017): Association of matrix metalloproteinase levels with collagen degradation in the context of abdominal aortic aneurysm. Eur. J. Vasc. Endovasc. Surg. 53, 549-558 https://doi.org/10.1016/j.ejvs.2016.12.030

Krishna SM, Dear AE, Norman PE, Golledge J (2010): Genetic and epigenetic mechanisms and their possible role in abdominal aortic aneurysm. Atherosclerosis 212, 16-29 https://doi.org/10.1016/j.atherosclerosis.2010.02.008
Lamblin N, Bauters C, Hermant X, Lablanche JM, Helbecque N, Amouyel P (2002): Polymorphisms in the promoter regions of MMP-2, MMP-3, MMP-9 and MMP-12 genes as determinants of aneurysmal coronary artery disease. J. Am. Coll. Cardiol. 40, 43-48 https://doi.org/10.1016/S0735-1097(02)01909-5

Li T, Lv Z, Jing J-J, Yang J, Yuan Y (2018): Matrix metalloproteinase family polymorphisms and the risk of aortic aneurysmal diseases: A systematic review and meta-analysis. Clin. Genet. 93, 15-32 https://doi.org/10.1111/cge.13050

Longo GM, Xiong W, Greiner TC, Zhao Y, Fiotti N, Baxter BT (2002): Matrix metalloproteinases 2 and 9 work in concert to produce aortic aneurysms. J. Clin. Invest. 110, 625-632 https://doi.org/10.1172/JCI0215334

Michel J-B, Martin-Ventura J-L, Egido J, Sakalihasan N, Treska V, Lindholt J, Allaire E, Thorsteinsdottir U, Cockerill G, Swedenborg J; FAD EU consortium (2011): Novel aspects of the pathogenesis of aneurysms of the abdominal aorta in humans. Cardiovasc. Res. 90, 18-27 https://doi.org/10.1093/cvr/cvq337

Mikołajczyk-Stecyna J, Korcz A, Gabriel M, Pawlaczyk K, Oszkinis G, Słomski R (2015): Gene polymorphism -418 G/C of tissue inhibitor of metalloproteinases 2 is associated with abdominal aortic aneurysm. J. Vasc. Surg. 61, 1114-1119 https://doi.org/10.1016/j.jvs.2013.12.045

Morris DR, Biros E, Cronin O, Kuivaniemi H, Golledge J (2014): The association of genetic variants of matrix metalloproteinases with abdominal aortic aneurysm: a systematic review and meta-analysis. Heart 100, 295-302

https://doi.org/10.1136/heartjnl-2013-304129

Ogata T, Shibamura H, Tromp G, Sinha M, Goddard KA, Sakalihasan N, Limet R, MacKean GL, Arthur C, Sueda T, et al. (2005): Genetic analysis of polymorphisms in biologically relevant candidate genes in patients with abdominal aortic aneurysms. J. Vasc. Surg. 41, 1036-1042 https://doi.org/10.1016/j.jvs.2005.02.020

Petersen E, Boman J, Wågberg F, Angquist KA (2002): Presence of Chlamydia pneumoniae in abdominal aortic aneurysms is not associated with increased activity of matrix metalloproteinases. Eur. J. Vasc. Endovasc. Surg. 24, 365-369 https://doi.org/10.1053/ejvs.2002.1726

Powell JT (2006): Genes predisposing to rapid aneurysm growth. Ann. NY Acad. Sci. 1085, 236-241 https://doi.org/10.1196/annals.1383.042

Sakalihasan N, Delvenne P, Nusgens BV, Limet R, Lapière CM (1996): Activated forms of MMP2 and MMP9 in abdominal aortic aneurysms. J. Vasc. Surg. 24, 127-133 https://doi.org/10.1016/S0741-5214(96)70153-2

Shi G-P and Lindholt JS (2013): Mast cells in abdominal aortic aneurysms. Curr. Vasc. Pharmacol. 11, 314-326 https://doi.org/10.2174/1570161111311030006

Shi ZG, Li JP, Shi LL, Li X (2012): An updated patent therapeutic agents targeting MMPs. Recent Pat. Anticancer Drug Discov. 7, 74-101 https://doi.org/10.2174/157489212798357976

Smallwood L, Allcock R, van Bockxmeer F, Warrington N, Palmer LJ, Iacopetta B, Golledge J, Norman PE (2008): Polymorphisms of the matrix metalloproteinase 9 gene and abdominal aortic aneurysm. Br. J. Surg. 95, 1239-1244 
https://doi.org/10.1002/bjs.6345

Tamarina NA, McMillan WD, Shively VP, Pearce WH (1997): Expression of matrix metalloproteinases and their inhibitors in aneurysms and normal aorta. Surgery 122, 264-272 https://doi.org/10.1016/S0039-6060(97)90017-9

Thompson AR, Drenos F, Hafez H, Humphries SE (2008): Candidate gene association studies in abdominal aortic aneurysm disease: a review and meta-analysis. Eur. J. Vasc. Endovasc. Surg. 35, 19-30 https://doi.org/10.1016/j.ejvs.2007.07.022

Thompson RW, Geraghty PJ, Lee JK (2002): Abdominal aortic aneurysms: basic mechanisms and clinical implications. Curr. Probl. Surg. 39, 110-230 https://doi.org/10.1067/msg.2002.121421

Untergasser A, Cutcutache I, Koressaar T, Ye J, Faircloth BC, Remm M, Rozen SG (2012): Primer3--new capabilities and interfaces. Nucleic Acids Res. 40, e115 https://doi.org/10.1093/nar/gks596

van ,t Hof FNG, Ruigrok YM, Lee CH, Ripke S, Anderson G, de Andrade M, Baas AF, Blankensteijn JD, Böttinger EP, Bown MJ, et al. (2016): Shared genetic risk factors of intracranial, abdominal, and thoracic aneurysms. J. Am. Heart Assoc. 5, e002603
https://doi.org/10.1161/JAHA.115.002603

Visse R and Nagase H (2003): Matrix metalloproteinases and tissue inhibitors of metalloproteinases: structure, function, and biochemistry. Circ. Res. 92, 827-839 https://doi.org/10.1161/01.RES.0000070112.80711.3D

Wahlgren CM, Larsson E, Magnusson PKE, Hultgren R, Swedenborg J (2010): Genetic and environmental contributions to abdominal aortic aneurysm development in a twin population. J. Vasc. Surg. 51, 3-7 https://doi.org/10.1016/j.jvs.2009.08.036

Wei C-H, Phan L, Feltz J, Maiti R, Hefferon T, Lu Z (2018): tmVar 2.0: integrating genomic variant information from literature with dbSNP and ClinVar for precision medicine. Bioinformatics 34, 80-87 https://doi.org/10.1093/bioinformatics/btx541

Yasmin, O'Shaughnessy KM (2008): Genetics of arterial structure and function: towards new biomarkers for aortic stiffness? Clin. Sci. (Lond) 114, 661-677 https://doi.org/10.1042/CS20070369

Received: August 14, 2019

Final version accepted: October 8, 2019 